\title{
$X$-ray microtomography to study the microstructure of cream cheese-type products
}

\author{
J. Laverse, ${ }^{\star}$ M. Mastromatteo, $†$ P. Frisullo, ${ }^{\star}$ and M. A. Del Nobile ${ }^{\star} \ddagger^{1}$ \\ *Department of Food Science, \\ †Department of Production and Innovation in Mediterranean Agriculture and Food System (PrIME), and \\ łIstituto per la Ricerca e le Applicazioni Biotecnologiche per la Sicurezza e la Valorizzazione dei Prodotti Tipici e di Qualità, Università degli Studi \\ di Foggia, BIOAGROMED - Via Napoli, 52 - 71100 Foggia, Italy
}

\begin{abstract}
In this work, the imaging x-ray microtomography technique, new to the field of food science, was used for the analysis of fat microstructure and quantification of the fat present in cream cheese-type products. Five different types of commercially produced cheeses, chosen for their variability of texture, were used for this experiment: sample A, sample B, sample C, sample D, and sample E. Appropriate quantitative 3-dimensional parameters describing the fat structure were calculated (e.g., the geometric parameter percentage of fat volume was calculated for each image as a representation of the percentage of total fat content within the sample). The dynamic-mechanical properties of these samples were also studied using a controlled-strain rotational rheometer. Storage modulus and loss modulus were determined in a frequency range of 0.01 to $10 \mathrm{~Hz}$. The strain value was obtained by preliminary strain sweep oscillatory trials to determine the linear viscoelastic region of the cream cheese-type products. Statistical correlation analysis was performed on the results to help identify any microstructural-mechanical structure relationships. The results from this study show that microtomography is a suitable technique for the microstructural analysis of fat in cream cheese-type products, as it does not only provide an accurate percentage of the volume of the fat present but can also determine its spatial distribution.
\end{abstract}

Key words: x-ray microtomography, microstructure, cream cheese-type products

\section{INTRODUCTION}

Cream cheese is a spreadable soft cheese that structurally differs from other cheeses because of its lack of

Received June 11, 2010.

Accepted September 25, 2010.

${ }^{1}$ Corresponding author: ma.delnobile@unifg.it a compact protein matrix, coupled with relatively high moisture content. Its major structural component is fat (approximately 33\%), in the form of clusters of globules interspersed within milk proteins (Kaláb, 1993); its microstructure has been defined as corpuscular, or composed of compact fat casein aggregates with large spaces filled with whey (Kaláb and Jodler, 1985). The sensory and texture characteristics of commercially processed cream cheeses sold in the market today vary significantly. The influences of various design parameters on the sensory attributes of cream cheeses have been investigated and relationships between fat content, processing conditions, and sensory and structural properties have been established in different model systems (Langton et al., 1997, 1999; Wendin et al., 1997a, b). Structure-property relationships can strongly affect the physiochemical, functional, technological, and even nutritional properties of foods. For example, with regard to cream cheese-type products, the consumer's appreciation of these products is strongly linked to the texture. For texture, sensory properties are related to both mechanical properties and cellular structure. From this point of view, rheological and microstructural properties of cream cheese are particularly important and influence to a large extent the physical, textural, and sensory properties. Developing a proper understanding of the microstructure, particularly the spatial distribution and interaction of food components, is a key tool in developing cream cheeses with desired textural and organoleptic properties. In this context, determining the relationships between a given rheological property and the cellular structure is, thus, of prime importance.

In recent years, information about the 3-dimensional microstructure and ingredients of semi-solid foods such as cream cheeses can now be obtained using various imaging techniques. Although a wide variety of imaging techniques exist, they are mostly invasive, as they require sample preparation; hence, they cause the formation of artifacts. X-ray microtomography $(\boldsymbol{\mu} \mathbf{C T})$, on the other hand, is a noninvasive and nondestructive technique that has several advantages over other methods, includ- 
ing the ability to image low-moisture materials. X-ray microtomography uses the differences in x-ray attenuation, arising, principally, from differences in density within the specimen. A series of 2-dimensional x-ray images are obtained as a sample is rotated. The series of slices, covering the entire sample, can be rendered into a 3-dimensional image that can be presented either as a whole or as virtual slices of the sample at different depths and in different directions. Manipulation and analyses of $\mu \mathrm{CT}$ data using special software also allows reconstruction of cross-sections at depth increments as low as $15 \mu \mathrm{m}$, and along any desired orientation of the plane of cut. A series of noninvasive $\mu \mathrm{CT}$ slices of the same sample in any direction can provide much more information than just one scanning electron microscopy or optical imaging picture, for example.

Given the enormous success of $\mu \mathrm{CT}$ in medical applications, material science, chemical engineering, geology, and biology, it is not surprising that in recent years much attention has been focused on extending this imaging technique to food science, as a useful technique to aid in the study of food microstructure. This technique has been successfully used to observe the stability of gas bubbles in dough during the breadmaking process (Whitworth and Alava, 1999), the microstructure of foams (Lim and Barigou, 2004), 3-dimensional quantitative analysis of breadcrumbs (Falcone et al., 2005), the study of bread porous structure (Falcone et al., 2004), and ice crystals within frozen foods (Mousavi et al., 2005). Recently, this technique has also been used to study the bubble size distribution in wheat flour dough (Bellido et al., 2006), the effect of far-infrared radiation-assisted drying on microstructure of banana slices (Léonard et al., 2008), 3-dimensional pore space quantification of apple tissue (Mendoza et al., 2007) and the role of sugar and fat in sugar-snap cookies (Pareyt et al., 2009). X-ray microtomography provides in-depth information on the microstructure of the food product being tested; therefore, a better understanding of the physical structure of the product and, from an engineering perspective, knowledge about the microstructure of foods can be used to identify the important processing parameters that affect the quality of a product. Processes are no longer designed from a macroscopic level; knowing the properties of foods on the micro scale determines the process specification. Hence, $\mu \mathrm{CT}$ is fast becoming a very useful tool to aid in the study of food microstructure.

The aim of this article is to demonstrate the capability of $\mu \mathrm{CT}$ as a useful tool for the quantification of the microstructure in dairy food products such as cream cheese and, furthermore, to characterize the influences of fat content on microstructure of cheese spreads and its relation to cheese spread rheology.

\section{MATERIALS AND METHODS}

\section{Raw Materials}

Five different types of commercially produced cream cheese-type products, chosen for their variability of texture, were used for this experiment: sample A, sample B, sample C, sample D, and sample E. They were purchased locally and all tests, microstructural and rheological, were carried out on the same day. Three samples were prepared for each type of cheese, each $28 \mathrm{~mm}$ in diameter with a thickness of $18 \mathrm{~mm}$. Each sample used for $\mu \mathrm{CT}$ analysis was wrapped with parafilm to avoid dispersion of moisture; the parafilm did not interfere with the x-rays.

\section{Tomographical Analysis}

For $\mu \mathrm{CT}$, the samples were imaged under the same conditions, using the Skyscan 1172 high-resolution desktop x-ray microtomography system (Skyscan, Kontich, Belgium). A cheese sample was placed on a cylindrical plate; the source and the detector were fixed, while the sample was rotated during measurement. Power settings of $100 \mathrm{kVp}$ and $100 \mu \mathrm{A}$ were used. A chargedcoupled device camera with $2000 \times 1048$ pixels was used to record the transmission of the conical x-ray beam through all samples. The distance source-objectcamera was adjusted to produce images with a pixel size of $2 \mu \mathrm{m}$. Four-frame averaging, a rotation step of $0.40^{\circ}$, and an exposure time of $1,767 \mathrm{~ms}$ were chosen to minimize the noise, covering a view of $180^{\circ}$. Smoothing and beam-hardening correction steps were applied to suppress noise and beam-hardening artifacts, respectively. Beam-hardening correction was only moderately applied, set to $25 \%$ within NRecon reconstruction software (Skyscan), and a fast ring artifacts reduction (set to 51 within NRecon) was also applied. Once initial parameters were set, the acquisition step was completely automated and did not require operator assistance. Scan time, on average, required $30 \mathrm{~min}$. A set of flat cross section images, was obtained for each sample after tomographical reconstruction by the reconstruction software NRecon (Skyscan). Three-dimensional reconstructions of samples were created by effectively stacking all 2-dimensional tomographs, a total of 125 slice images with a slice spacing of $0.069 \mathrm{~mm}$.

For image processing and analysis, the Skyscan software, Computed Tomography-Analyzer (CTAn) was used. Image segmentation was first carried out on the smoothed 8-bit gray-scale images obtained from the reconstruction step, using CTAn (Skyscan) software. Segmentation is the process of converting the grayscale images into black and white images by assigning 
the value 1 to all pixels whose intensity was below a given gray tone value and 0 to all the others. For this, an automatic threshold based on the entropy of the histogram (Sahoo et al., 1988) was calculated for each image. The lower gray threshold (8) and upper gray threshold (110) values were identified; each sample was processed under the same conditions.

For data analysis, before 3-dimensional reconstruction, a component-labeling algorithm, available within CTAn, was used to isolate the largest 3-dimensional connected structures. All reconstructions where created in CTAn using an adaptive rendering (locality 10 and tolerance 0.25) algorithm and saved as P3G surface models (SkyScan model format). P3G models were then imported into CT vol software (Skyscan) for visualization.

The following 7 geometric parameters were measured using the CTAn software (Skyscan): percentage of object (i.e., fat) volume (POV), object surface/volume ratio (OSVR), fragmentation index (FI), structure thickness (ST), structure separation (SS), structure modeling index (SMI), and degree of anisotropy (DA). The POV is the proportion of the volume of interest occupied by binarized solid objects (i.e., fat). The OSVR is the basic parameter in characterizing the complexity of the structures and is also the basis of model-dependent estimates of thickness (i.e., size and distribution of fat present in each sample). The FI is an index of connectivity of structure, which was developed and defined by Hahn et al. (1992); it calculates the index of relative convexity or concavity of the total surface of the sample. Structure thickness calculates or estimates the true structure thickness of the sample from 2-dimensional measurements. The SS is essentially the thickness of the spaces as defined by binarization within the volume of interest (i.e., fat). It can also be calculated either from 2-dimensional images with model assumptions or directly in 3 dimensions. The SMI estimates the characteristic form of which the structure is composed (i.e., whether it is more plate-like, rod-like, or even sphere-like; $0=$ ideal plate, $3=$ cylinder, and 4 $=$ sphere). Finally, the DA is a measure of preferential alignment of fat.

\section{Rheological Analyses}

The dynamic-mechanical properties of the 5 cream cheese samples were studied using a controlled-strain rotational rheometer (ARES model, TA Instruments, New Castle, DE) equipped with a force rebalance transducer (model 1K-FRTN1, 1-1,000 g cm, $200 \mathrm{rad} / \mathrm{s}, 2-2,000$ gram force) and parallel plates (superior plate diameter of $50 \mathrm{~mm}$ ). A steady temperature was ensured with an accuracy of $\pm 0.1^{\circ} \mathrm{C}$ by means of a controlled fluid bath unit and an external thermostatic bath. Three samples were prepared for each type of cheese, each weighing 7 $\mathrm{g}$; the samples were placed onto the surface of the lower plate and the upper plate was lowered until it reached a 2-mm gap distance to avoid sample disruption, and the excess sample was trimmed. To prevent water evaporation, a suitable cover tool sealing the top of the superior plate was used during testing. Storage modulus $\left(\mathbf{G}^{\prime}\right)$ and loss modulus $\left(\mathbf{G}^{\prime \prime}\right)$ were determined in a frequency range of 0.01 to $10 \mathrm{~Hz}$. The strain value was obtained by preliminary strain sweep oscillatory trials to determine the linear viscoelastic region. The strain sweep oscillatory tests were carried out at a frequency of 1 $\mathrm{Hz}$ and in a range of shear strain of 0.01 to $99 \%$. All experiments were carried out at $4^{\circ} \mathrm{C}$. Three repetitions of the dynamic-mechanical experiments were performed for each cream cheese sample.

\section{Statistical Analysis}

Data were subjected to ANOVA, using the SPSS statistical software (1999; IBM SPSS Statistics Professional). The mathematical model for $\mu \mathrm{CT}$ parameters and rheological parameters included fixed effects. When significant effects were found (at $P<0.05$ ), the Student's $t$-test was used to locate significant differences between means. Pearson correlation coefficients (CORR procedure of SPSS) were calculated to examine the relationship between the rheological parameters and all $\mu \mathrm{CT}$ parameters.

\section{RESULTS AND DISCUSSION}

The results of the $\mu \mathrm{CT}$ analysis were used to study the total fat microstructure. Furthermore, the additional information gained from the $\mu \mathrm{CT}$ analysis (i.e., the geometric parameters mentioned above), provided the required information to characterize and investigate the correlation, if any, among the microstructures and the rheological properties of the different types of cheeses.

\section{Total Fat Microstructure}

Figure 1 shows the gray level reconstructed cross section images of the samples acquired by $\mu \mathrm{CT}$; the contrasts in these images are based on the differences in absorption of x-rays by the constituents of the sample (e.g., fat, protein, and air). This contrast is produced by a variation of density and a change in composition of the sample and is based exclusively on the detection of an amplitude variation of $\mathrm{x}$-rays transmitted through the sample itself. The obtained image is a map of the spatial distribution of the x-rays in which the brighter regions correspond to the higher level of attenuation 


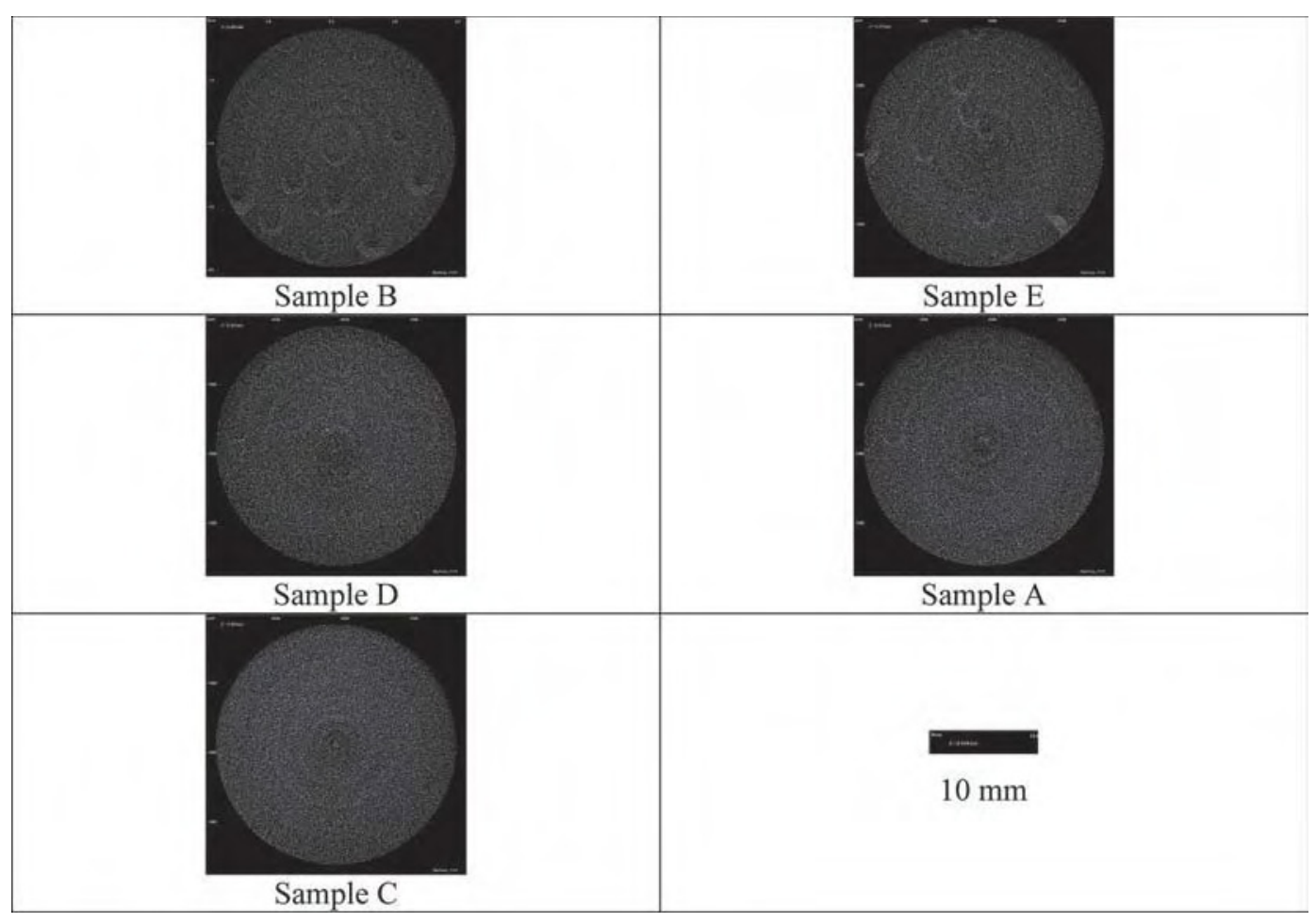

Figure 1. Gray-scale tomography of cream cheese samples where the white objects are fat and protein. The brighter objects are fat and are represented in Figure 2.

(i.e., higher density region). As fat has a higher absorption coefficient with respect to protein, the fat phase is easily visualized as the brighter objects in Figure 1. Further information cannot be acquired from these images, as these are only 2-dimensional representations of the samples. On the other hand, Figure 2 shows the 3 -dimensional reconstructions for each sample from which the geometrical parameters were calculated using the CTAn software (Skyscan). From these images, it can be noted that sample B and sample E are very similar in terms of structure distribution. The structures present in these samples appear to be large unevenly distributed clusters. The geometric parameter POV was calculated for each image as an estimation of the percentage of total fat content within the sample. This estimation was verified by comparing the POV values to that of the manufacturer's stated fat content (see Table 1). This table confirms that the POV can indeed represent the estimated fat content present as stated by the manufacturer.

Table 2 shows the average values obtained for the following 7 microstructural parameters using the CTAn software (Skyscan): POV, OSVR, FI, ST, SS, SMI, and DA. The results came from the statistical analysis carried out as reported above. The FI is the index of connectivity and is a measure of relative convexity or concavity of the total solid surface, based on the prin- ciple that concavity indicates connectivity, and convexity indicates isolated disconnected structures (Lim and Barigou, 2004). A lower FI signifies more connected solid lattices and has a negative index; on the other hand, a higher FI indicates a more disconnected solid structure and has a positive index. As can be noted from the results, the FI is negative for all samples; therefore, the fat structures in these samples have more connected solid lattices and, therefore, are concave in structure. The DA is a measure of the 3-dimensional structural symmetry (i.e., in this case, it indicates the presence or absence of preferential alignment of the fat present along a particular direction; Lim and Barigou, 2004). A value of 0 would correspond to total isotropy, whereas a value of 1 would indicate total anisotropy. According

Table 1. Percentage of fat measured by the x-ray microtomography technique and the manufacturer's stated fat content

\begin{tabular}{lcc}
\hline Sample & POV $^{1}$ & $\begin{array}{c}\text { Manufacturer's } \\
\text { value }(g)\end{array}$ \\
\hline Sample B & 28.68 & 25.5 \\
Sample E & 30.60 & 31.5 \\
Sample C & 15.49 & 16.5 \\
Sample A & 20.20 & 19.6 \\
Sample D & 26.96 & 26.5 \\
\hline
\end{tabular}

${ }^{1}$ Percentage of volume. 
Table 2. Values of the geometric parameters ${ }^{1}$ for cream cheese samples

\begin{tabular}{|c|c|c|c|c|c|c|c|}
\hline Sample & POV & OSVR & FI & $\mathrm{ST}$ & SS & $\mathrm{DA}$ & SMI \\
\hline Sample B & $28.68^{\mathrm{a}}$ & $0.18^{\mathrm{a}}$ & $-0.21^{\mathrm{a}}$ & $34.52^{\mathrm{a}}$ & $27.59^{\mathrm{a}}$ & 0.73 & $2.62^{\mathrm{a}}$ \\
\hline Sample E & $30.60^{\mathrm{a}}$ & $0.16^{\mathrm{b}}$ & $-0.24^{\mathrm{a}}$ & $35.12^{\mathrm{a}}$ & $26.89^{\mathrm{a}}$ & 0.73 & $2.62^{\mathrm{a}}$ \\
\hline Sample C & $15.49^{\mathrm{b}}$ & $0.25^{\mathrm{c}}$ & $-0.25^{\mathrm{a}}$ & $26.32^{\mathrm{b}}$ & $22.21^{\mathrm{b}}$ & 0.80 & $2.57^{\mathrm{a}}$ \\
\hline Sample D & $26.96^{\mathrm{d}}$ & $0.25^{\mathrm{c}}$ & $-0.21^{\mathrm{a}}$ & $34.75^{\mathrm{a}}$ & $18.82^{\mathrm{d}}$ & 0.88 & $2.42^{\mathrm{a}}$ \\
\hline
\end{tabular}

${ }^{\mathrm{a}-\mathrm{d}}$ Means in the same column followed by different superscript letters differ significantly $(P<0.05)$.

${ }^{1} \mathrm{POV}=$ percentage of volume; OSVR $=$ object surface/volume ratio; FI = fragmentation index; ST $=$ structure thickness; SS $=$ structure separation; DA = degree of anisotropy; SMI = structure modeling index. All parameters underwent one-way ANOVA and Duncan's test $(P<0.05)$ using Statistica for Windows software (StatSoft, Tulsa, OK).

to the results obtained for DA (see Table 1), the fat present in all samples are anisotropic and no statistical differences were found among the samples; this is in accordance with Fenoul et al. (2008), who stated that cream cheese has an anisotropic structure.

The POV is the percentage of the total fat content present in the sample. It can be seen that sample B and sample $\mathrm{E}$ have the highest and statistically equal POV values; therefore, these 2 samples are similar in terms of fat content. On the other hand, sample A, sample $\mathrm{C}$, and sample D have lower POV values. The OSVR indicates the fat distribution within the sample and depends on the homogenization pressure during manufacturing. The higher the OSVR value, the more finely distributed is the fat droplet present in the sample. It can be observed from the table that sample A, sample

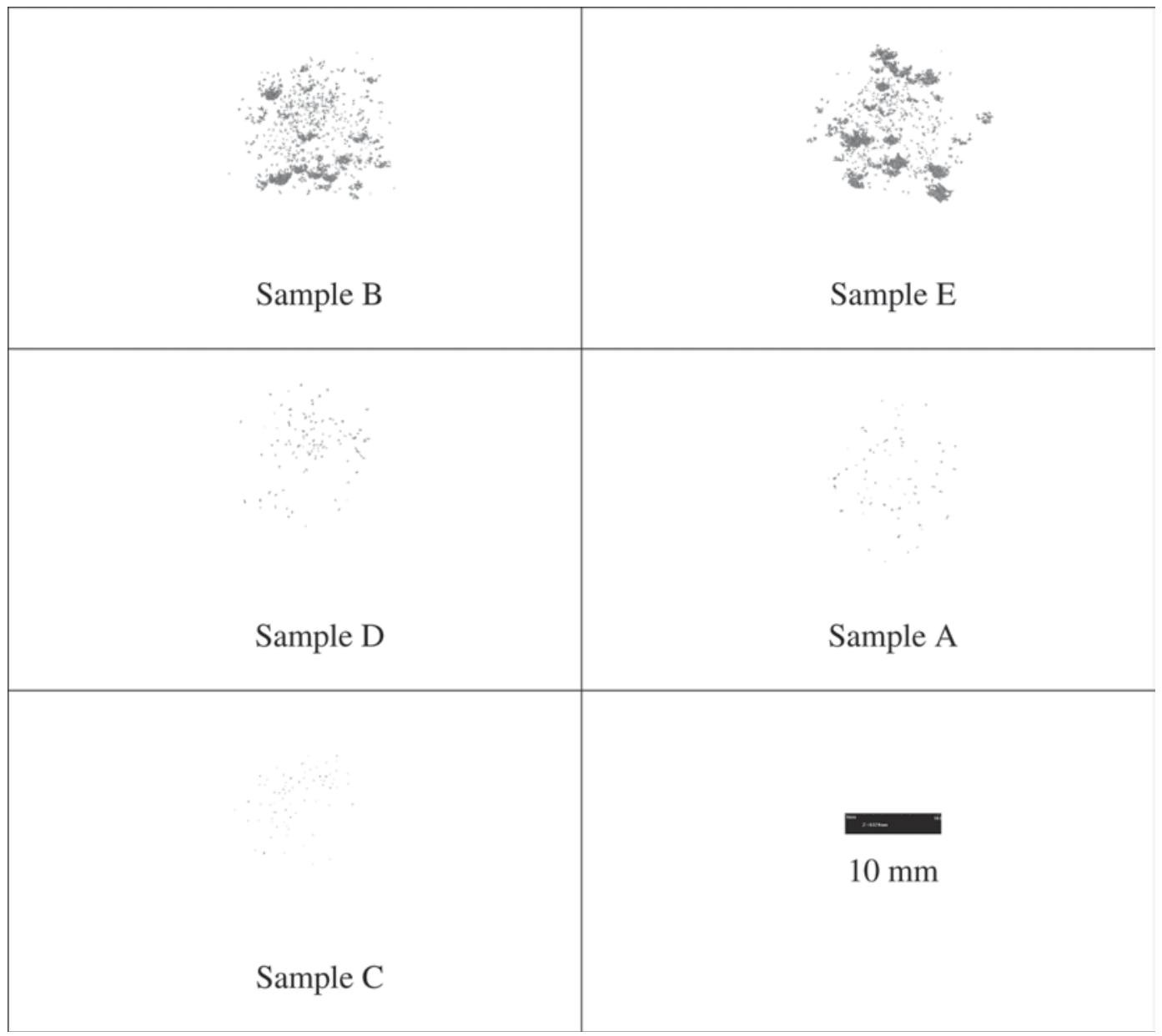

Figure 2. Three-dimensional reconstruction of the fat present in the cream cheese samples using x-ray microtomography ( $\mu \mathrm{CT})$. 
Table 3. The coordination number $z$ and the coefficient $A$ values for cream cheese samples ${ }^{1}$

\begin{tabular}{lcccc}
\hline Sample & $A$ & $\mathrm{~d} A^{2}$ & $z$ & $\mathrm{~d} z^{2}$ \\
\hline Sample A & $4,811.25$ & 4.65 & 9.05 & $7.11 \mathrm{e}-2$ \\
Sample B & 826.41 & 2.65 & 8.56 & 0.21 \\
Sample C & $1,579.48$ & 4.38 & 7.17 & 0.13 \\
Sample D & 427.59 & 0.63 & 9.49 & 0.12 \\
Sample E & 692.91 & 1.44 & 8.92 & 0.15 \\
\hline${ }^{1}$ The coordination number $z$ gives the level of interactions of rheological units and the coefficient $A$ their sta- \\
bility. \\
${ }^{2}$ d $=$ standard deviation.
\end{tabular}

$\mathrm{C}$, and sample $\mathrm{D}$ have the highest and statistically equal values, hence having a much finer dispersion of oil droplets as compared with that of the other 2 samples. Although sample B and sample E have the lowest OSVR values, they are also statistically equal; therefore, these samples also have approximately the same type of fat distribution. Their low values indicate that the fat droplets present in these samples are more largely distributed compared with those in the other 3 samples. This is supported by Wendin et al. (2000), who found that an increase in fat content resulted in an increase in droplet size, and by Mistry and Anderson (1993), Desai and Nolting (1995), and Bryant et al. (1995), who showed that in full-fat cheeses clustering was prominent and the fat globules were of variable size and shape. The ST is the average thickness of the fat structure present; this parameter calculates a volume-based thickness of the structure in 3 dimensions independent of an assumed structure type. It can be noted from Table 2 that the samples with the highest fat content, sample B, sample D, and sample E have the highest ST values that are also statistically equal; therefore, these samples have similar fat thickness. The SS is the average distance between the fat structures in the samples. It can be noted from this table that sample B and sample E have the highest and statistically equal values for the SS parameter; this could be due to their fat droplets being in clusters and unevenly distributed. On the other hand, sample A, sample C, and sample D have the lowest, although not statistically equal, SS values; this could be due to their much finer dispersion of fat droplets compared with that of the other 2 samples.

No significant differences were found for the SMI in the different cream cheese samples. The SMI parameter; that is, a topological index, gives an estimate of the characteristic shape of a structure in terms of plates and cylinders composing the 3-dimensional structure (Hildebrand and Rüegsegger, 1997) and is calculated using a differential analysis of triangulated surface of the structure under examination. The SMI assumes integer values of 0,3 , and 4 for ideal plates, cylinders, and spheres, respectively. The SMI values calculated for all of the samples ranged from 2.62 to 2.42. These values are fairly close to 3 , therefore suggesting that the characteristic shape of the fat droplets present in cream cheese are cylindrical in nature.

\section{Rheological Analysis}

Figure 3 shows the rheological data, $\mathrm{G}^{\prime}$ and $\mathrm{G}^{\prime \prime}$ values vs. the oscillatory frequency for the 5 cream cheese samples. It can be inferred from this figure that all samples exhibited viscoelastic behavior with $\mathrm{G}^{\prime}$ greater than $\mathrm{G}^{\prime \prime}$ (Rao and Steffe, 1992; Subramanian and Gunasekaran, 1997). It can be noted in the results of Figure 3 that the samples with the highest fat content, sample B, sample $\mathrm{E}$, and sample $\mathrm{D}$ have the lowest values of $\mathrm{G}^{\prime}$.

The experimental data on the frequency sweep tests were correlated using the following power law according to Bohlin's theory of flow as a cooperative phenomenon (Bohlin, 1980):

$$
\mathrm{G}^{\prime}=A \cdot \omega \cdot \exp (1 / z)
$$

where $\omega$ is the frequency. On the basis of this theory, emulsions are modeled as a network of rheological units that interact for establishing system structure. The coordination number $z$ gives the level of these interactions and the coefficient $A$ their stability. The $z$ and $A$ values were obtained for all samples and are shown in Table 3. These parameters give an idea of the emulsion stability. It can be noted from Table 3 that sample A, sample B, and sample E show statistically similar values for the $z$ parameter and that sample $\mathrm{D}$ has the highest value with respect to all of the other samples. The high $z$ value of sample D indicates a more complex structure and, therefore, more microstructural interactions with respect to the other samples. Regarding the $A$ parameter, all samples differ significantly. Moreover, the highest and lowest $A$ values were recorded for sample A and sample D, respectively; therefore, sample A is more stable (Peressini et al., 1998) with respect to sample E. 
Table 4. Correlation results for all of the microstructural and rheological parameters ${ }^{1}$ of cream cheese samples

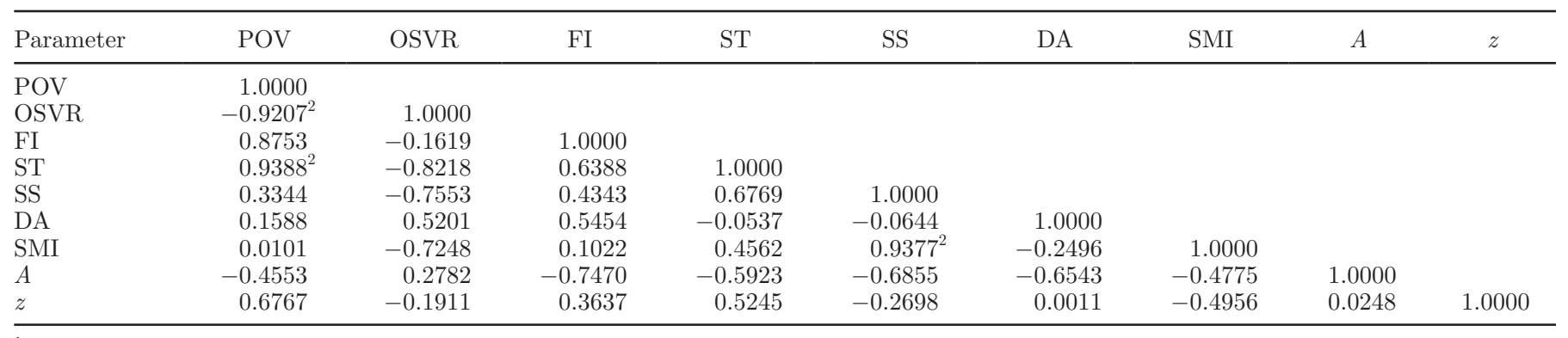

${ }^{1} \mathrm{POV}=$ percentage of volume; OSVR $=$ object surface/volume ratio; FI $=$ fragmentation index; $\mathrm{ST}=$ structure thickness; SS $=$ structure separation; $\mathrm{DA}=$ degree of anisotropy; $\mathrm{SMI}=$ structure modeling index; $A=$ stability coefficient; $z=$ coordination number.

${ }^{2}$ Highly, positively, and significantly correlated.

\section{Correlation}

Table 4 shows the results of the correlation among the microstructural parameters and rheological properties of the samples. It can be noted from this table that no statistically significant correlations $(P<0.1)$ were found among the microstructural and rheological parameters measured but correlation exists among some of the following microstructural properties: POV, OSVR, ST, SMI, and SS. The correlation that exists among the parameters POV, OSVR, and ST is in agreement with that found by Wendin et al. (2000), Mistry and Anderson (1993), Desai and Nolting (1995), and Bryant et al. (1995), who found that fat content was related to droplet size and distribution.

The lack of correlation among the rheological parameters and the fat microstructure may be due to the fact that the rheological parameters are more correlated to the protein network than to the fat microstructure. This preliminary investigation will allow us to carry out further study on the microstructural properties and their effects on cream cheese texture. Future studies will also investigate the chemical composition of cream cheeses and its relationship to the microstructure and the rheology. This should aid in the optimization of the process variables that affect the characteristics of

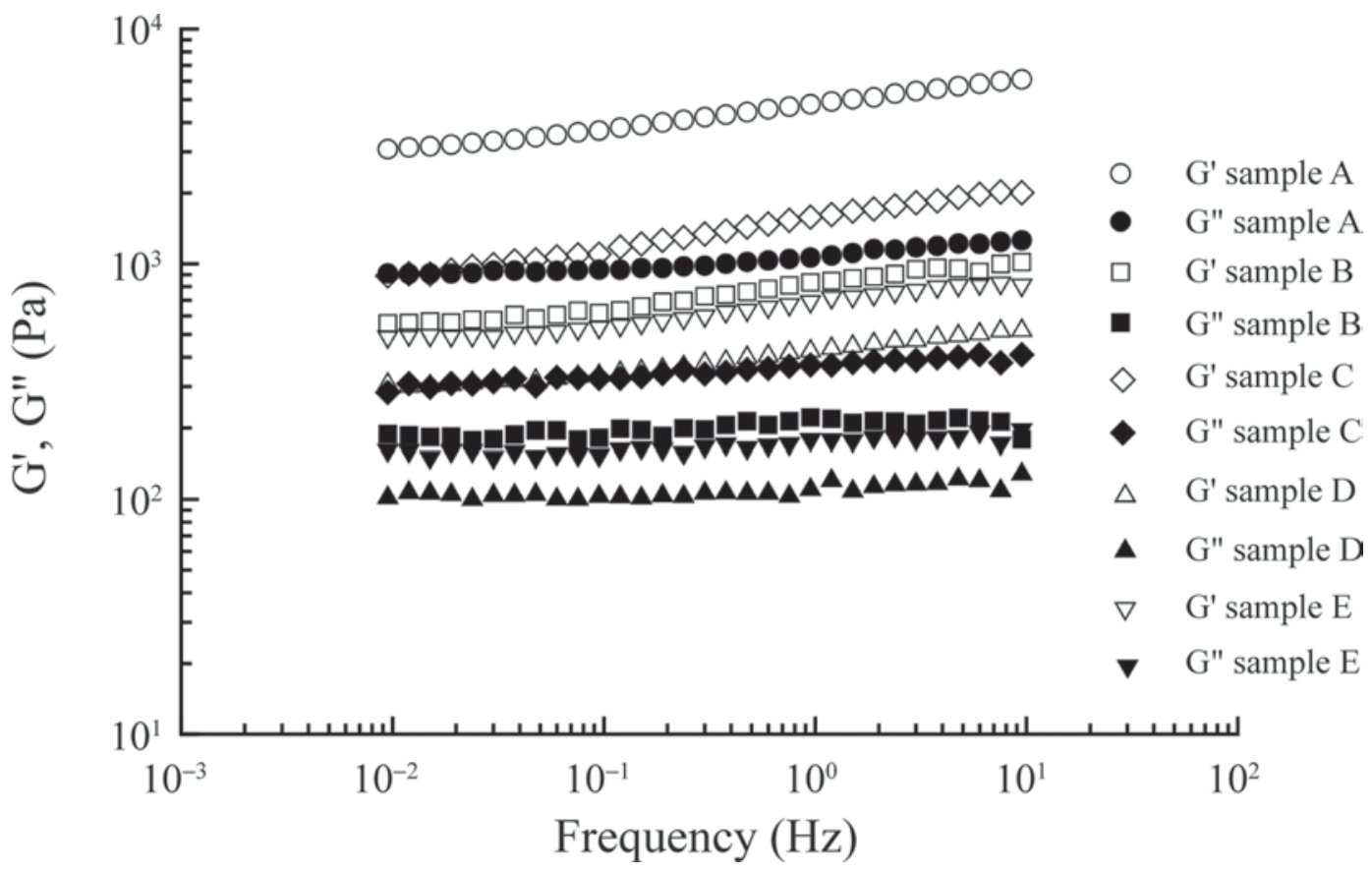

Figure 3. Rheological data, storage modulus ( $\mathrm{G}^{\prime}$; open symbols), and loss modulus ( $\mathrm{G}^{\prime \prime}$; solid symbols) values vs. the oscillatory frequency

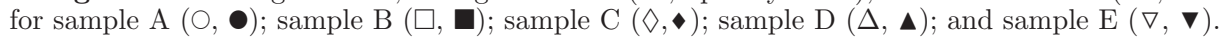


cream cheeses that are involved in consumer satisfaction to promote full product acceptability.

\section{CONCLUSIONS}

The microstructure of cream cheeses can be successfully analyzed using X-ray microtomography ( $\mu \mathrm{CT})$. Although the correlation performed on the $\mu \mathrm{CT}$ parameters to the rheological parameters did not identify any microstructural-mechanical structure relationships, relationships among the different microstructural parameters; that is, the relationship between fat content to droplet size and distribution, were identified. This is important as these relationships can affect the functional, technological, and even nutritional properties of foods. Although the correlation performed on the $\mu \mathrm{CT}$ parameters to the rheological parameters did not identify any microstructural-mechanical structure relationships, relationships among the different microstructural parameters (i.e., the relationship between fat content and droplet size and distribution) were identified. This is of importance, as these relationships can affect the functional, technological, and even nutritional properties of foods.

\section{REFERENCES}

Bellido, G. G., M. G. Scanlon, J. H. Page, and B. Hallgrimsson. 2006. The bubble size distribution in wheat flour dough. Food Res. Int. 39:1058-1066.

Bohlin, L. 1980. A theory of flow as a cooperative phenomenon. J. Colloid Interface Sci. 74:423-434.

Bryant, A., Z. Ustunol, and J. Steffe. 1995. Texture of Cheddar cheese as influenced by fat reduction. J. Food Sci. 60: 1216-1219.

Desai, N., and J. Nolting. 1995. Microstructure studies of reduced-fat cheeses containing fat substitute. Pages 295-302 in Chemistry of Structure-Function Relationships in Cheese. E. L. Malin and M. H. Tunick, ed. Plenum Press, New York, NY.

Falcone, P. M., A. Baiano, F. Zanini, L. Mancini, G. Tromba, D. Dreossi, and M. A. Del Nobile. 2005. Three-dimensional quantitative analysis of bread crumb by X-ray microtomohraphy. J. Food Sci. 70:265-272.

Falcone, P. M., A. Baiano, F. Zanini, L. Mancini, G. Tromba, F. Montanari, and M. A. Del Nobile. 2004. A novel approach to the study of bread porous structure: Phase-contrast X-ray micro-tomography. J. Food Sci. 69:38-43.

Fenoul, F., M. Le Denmat, F. Hamdi, G. Cuvelier, and C. Michon. 2008. Technical Note: Confocal scanning laser microscopy and quantitative image analysis: Application to cream cheese microstructure investigation. J. Dairy Sci. 91:1325-1333.

Hahn, M., M. Vogel, M. Pompesius-Kempa, and G. Delling. 1992. Trabecular bone pattern factor-A new parameter for simple quantification of bone microarchitecture. Bone 13:327-330.
Hildebrand, T., and P. Rüegsegger. 1997. A new method for the modelindependent assessment of thickness in three-dimensional images J. Microsc. 185:67-75.

Kaláb, M. 1993. Practical aspects of electron microscopy in dairy research. Food Structure. 12:95-114.

Kaláb, M., and H. W. Jodler. 1985. Milk gel structure. XV. Electron microscopy of whey protein-based Cream cheese spread. Milchwissenschaft 40:193-196.

Langton, M., A. Astrom, and A.-M. Hermansson. 1999. Microstructure in relation to the textural properties of mayonnaises. Pages 366-376 in Food Emulsions and Foams: Interfaces, Interactions and Stability. E. Dickinson and J. M. Rodriguez Patino, ed. The Royal Society of Chemistry, London, UK.

Langton, M., A. Astrom, M. Stading, and A.-M. Hermansson. 1997. Effect of microstructure on the sensory perception of particulate gels. Pages 18-28 in Food Colloids, Proteins, Lipids and Polysaccharides. E. Dickinson and B. Bergenstahl, ed. The Royal Society of Chemistry, London, UK.

Léonard, A., S. Blacher, C. Nimmol, and S. Devahastin. 2008. Effect of far-infrared radiation assisted drying on microstructure of banana slices: An illustrative use of X-ray microtomography in microstructural evaluation of a food product. J. Food Eng. 85:154-162.

Lim, K. S., and M. Barigou. 2004. X-ray micro-tomography of cellular food products. Food Res. Int. 37:1001-1012.

Mendoza, F., P. Verboven, H. M. Mebatsion, and G. Kerckhofs. 2007. Three-dimensional pore space quantification of apple tissue using X-ray computed microtomography. Planta 226:559-570.

Mistry, V. V., and D. L. Anderson. 1993. Composition and microstructure of commercial full fat and low-fat cheese. Food Structure 12:259-266.

Mousavi, R., T. Miri, P. W. Cox, and P. J. Fryer. 2005. A novel technique for ice crystal visualization in frozen solids using X-ray micro-computed tomography. J. Food Sci. 70:437-442.

Pareyt, B., F. Talhaoui, G. Kerckhofs, K. Brijs, H. Goesaert, M. Wevers, and J. A. Delcour. 2009. The role of sugar and fat in sugar-snap cookies: Structural and textural properties. J. Food Eng. 90:400-408.

Peressini, D., A. Sensidoni, and B. de Cindio. 1998. Rheological characterization of traditional and light mayonnaises. J. Food Eng. 35:409-417.

Rao, M. A., and J. F. Steffe. 1992. Viscoelastic Properties of Foods. Elsevier Applied Science, New York, NY.

Sahoo, P. K., D. W. Slaaf, and T. A. Albert. 1997. Threshold selection using a minimal histogram entropy difference. Opt. Eng. 36:1976-1981.

Subramanian, R., and S. Gunasekaran. 1997. Small amplitude oscillatory shear studies on Mozzarella cheese. Part I. Region of linear viscoelasticity. J. Texture Stud. 28:633-642.

Wendin, K., K. Aaby, A. Edris, M. Risberg-Ellekjaer, R. Albin, B. Bergenstahl, L. Johansson, E. Pilman-Willers, and R. Solheim. 1997a. Low-fat mayonnaise: Influences of fat content, aroma compounds and thickeners. Food Hydrocoll. 11:87-99.

Wendin, K., M. Langton, L. Caous, and G. Hall. 2000. Dynamic analyses of sensory and microstructural properties of cream cheese. Food Chem. 71:363-378.

Wendin, K., R. Solheim, T. Allmere, and L. Johansson. 1997b. Flavour and texture in sourmilk affected by thickeners and fat content. Food Qual. Prefer. 8:281-291.

Whitworth, M. B., and J. M. Alava. 1999. The imaging and measurement of bubble in bread doughs. Pages 221-231 in Bubbles in Foods. G.M. Campbell, C. Webb, S. S. Pandiella, and K. Niranjan, ed. Eagan Press, St. Paul, MN. 